\title{
Human-technology traffic jams (or harnessing human brainpower is more than a matter of logic)
}

\section{Sue McNamara Monash University}

\begin{abstract}
This paper acknowledges the innovative developments being undertaken in technology based instructional projects in Australia. However the focus here is on the 'parking problems' or more precisely the 'traffic jams': current and future difficulties in design and management of converging technologies in education. Previous papers have called for greater consideration of the learner. This paper addresses the need for consideration of human factors relating to design and management which will determine the success of technology based instruction in education and training, both now and in the future.
\end{abstract}

\begin{abstract}
About that traffic jam ...
Most adults have some basic knowledge of cars. We might only know that somewhere under the bonnet is an engine which makes it go or that a steering wheel directs the car and a key is needed to start the engine. As long as we can reach the pedals this knowledge will, theoretically at least, allow us to use the nation's road system.
\end{abstract}

Most adults are also familiar with the phenomena of' the traffic jam'. The majority of us have, at some time or other in our lives, been seated in a stationary vehicle, sandwiched between two like vehicles in a line stretching to the horizon, or as far as they eye can see (whichever is closer). It is a 'phenomenon' in the most pure sense; "a thing that appears or is perceived, especially a thing the cause of which is in question" (Concise Oxford Dictionary p1271). And like most phenomena, the traffic jam tests human endeavour to its fullest extent. 
Humans, finding themselves involved in such experiences, demonstrate individual differences in temperament and behaviour ranging from simple calm to uncontrollable rage. Traffic jams illicit any number of problem solving approaches from patient acceptance of one's lot to more innovative, if somewhat unconventional solutions such as taking one's vehicle over a four or five inch concrete median strip in order to join the flow of traffic in the opposite direction. Presumably the vehicle's undercarriage survives the ordeal.

For each traffic jam there is usually a logical explanation and an appropriate response, however this logical explanation and appropriate response are of little consolation to those involved in "a thing the cause of which is in question" and the end of which is nowhere in sight!

\section{Metaphorically speaking ...}

Australia has a complex and sophisticated road and traffic system. Yet in recent years we are hearing more and more complaints about the system's inability to cope with growth and development. Problems have been noted in both the areas of design and management. Traffic jams have become an accepted ordeal one must endure in the course of using the road system, particularly in areas of high volume traffic such as the freeways, highways, metropolitan and country roads. Even back lanes are not exempt as the daily battle for parking space intensifies.

The picture for the immediate and long term future is something of a double sided coin. On one side traffic control systems have at their disposal mechanisms such as the radar speed camera, red light camera and overhead speed indicators to assist traffic flow, while on the other side of the coin we appear to be witnessing a trend away from public transport, contributing to an escalating number of vehicles on our roads as cars become relatively cheaper (via the used car market) and more accessible.

\section{Traffic, technology and education}

In applying the metaphor of the traffic jam to human-technology interaction in education I would like to put the purpose of this paper in context with the words of Isaac Asimov (with acknowledgement to Christopher Dede who quoted them earlier this year);

What is important is not to forecast the automobile -- but rather the parking problem

There are a number of parallels which can be drawn between automobile traffic systems and technology in education and training. Converging technologies, multimedia and integrated technologies bring to mind the 
possibilities of numerous combinations of visual, aural, textual and other resources. To the designer they present the opportunity of making instruction more motivating, challenging, interesting and effective. To the system/course manager they suggest more efficient and effective instructional mechanisms: greater capacities for the flow and communication of information, administration and the opportunity to streamline management strategies and functions. A similar picture might be drawn for the road traffic system designer and manager.

There remains the other side of the coin. The synthesis and combinations of once discrete technologies is also cause for some rethinking on the part of education, training and in particular instructional design. Beneath the somewhat mesmerising and intriguing screen designs of the 'hyper-cybermulticonvergent-integrated instructional mechanism' lie the complexities and issues of design and management with which we in the world of education have to deal. We must parallel our enthusiastic acknowledgement of the potential of these developments with considerations of the implications for design and management of such systems.

\section{The human-technology traffic jam}

The dictionary defines 'traffic' as the 'passing or movement of vehicles or persons along a route' (p1138) and 'jam' as 'squeezing into a very right position' or, in relation to radio to 'send out signals which interfere with other signals on the same frequency' (p567 -- Heinemann Australian Dictionary). For the purposes of this discussion the second definition is perhaps more appropriate. If, using a little creative licence, we translate these definitions into the context of converging technologies and their application to education and training, we might define the 'human technology traffic jam' as a situation in which learning has been impeded due to signals interfering with the interaction between learner and learning. These signals might be related to design or management of the instructional system.

Dede (1990) provides some illustrations of human technology traffic jams in describing the transformation of technology. On the one hand some of us in education are at a stage in technological understanding of becoming rather familiar with the concepts of expert systems, artificial intelligence, hypermedia, cyberspace and such phenomena. On the other hand, as Dede points out; the larger proportion of society, and in our case learners, instructors, teachers, lecturers, trainers and so on are still at the stage of coming to grips with timing devices on video recorders and recognising a floppy disk. Further, in education we are seeing microworlds in which the rules of reality can be altered to explore the laws of physics (Dede, 1990), while at the same time we still have the panic-stricken user sitting for ages in front of a black screen before twenty seconds of help indicates that he 
needs to turn on the external hard drive of the Macintosh Plus in order to have a system operating! Such are the discrepancies between the realities of single project developments and the more general higher education implementation of convergent technologies.

The above suggests cautionary speed restrictions and the need for road signs in implementing the more sophisticated technology systems. It might be argued that such measures are unnecessary in view of the fact that convergence and integration are moving towards making the interface transparent but as Dede (1990) notes, we should perhaps be aware of myths and misconceptions which parallel moves in this direction. He describes four:

\section{The misconception or myth of 'consolidation'}

The notion that as standardisation becomes the more common practice all our computer problems will be resolved.

\section{The misconception or myth of 'literacy'}

Rather than focusing on computer literacy, information literacy, visual literacy and so on, we should go beyond the literacy metaphor to the establishment of a 'complementary cognitive relationship' between the human and technology.

\section{The misconception or myth of 'power' for the experts}

Power is needed by the novice perhaps more than by the expert. The greater the power the more the 'human' the capabilities of the technology and therefore the more appropriate for the novice.

\section{The misconception or myth of time}

The 'wait and see' mentality that encourages reactionary strategies rather than planned considerations of implementation.

In achieving a balance between technology exploration and implementation in education we need to parallel developments on a project level with an investment in research and planning for the more general application of the technologies in our educational and training institutions and organisations. Within this balancing exercise we must address the needs for such components as familiarisation training, support and backup (both technical and instructional) and budgetry planning for technology based learning.

A brief tour of some of the unique geographical and social features of current practices of technology in education illustrates points at which such driver assistance as tourist guides and information booths, road maps, safety islands and speed zones might cater to the wide variety of individuals touring, on business trips or settling into the world of convergent technological applications to education. 


\section{Designer traffic jams ...}

Jones (1989) maintains that "designers who are both psychologists and computer experts, scientists and visual artists are rare indeed" (p5), yet the requirements for design of multimedia and integrated technology systems and materials demands considerable expertise in each of these areas. Each of the areas is a specialist field in its own right and each contains sub-unit specialities. Within the computing area of technology based instructional systems lie the components of the operating system, file management and programming languages. The more complicated the system the greater the expertise required. Similarly, the psychological contributions to the design might specifically relate to the interpretation of learning theories, or they may overlap with the specialisations of the visual design areas of perception, graphic art and screen interface design. Engineering components of the input-output devices and the systems requirements of telecommunications specifications increases the complexity of the design process.

It is generally accepted that central to the development of materials and courseware based on convergent technology is a knowledge and understanding of both computer and human capabilities. The argument however is incomplete without equal consideration being given to the aural and visual components of the materials. While computer specifications and instructional systems design may dictate functions in making the materials operational, "the video script is a roadmap for the entire production. It is synonymous with program design" (Arwady and Gayeski, 1989 p9). Without due consideration to the 'artistry of the medium, particularly given the trends in associated industries such as the world of commercial entertainment, instructional materials development runs the risk or being unable to compete with more attractive and innovative uses of the technologies. Thus the traditional production components of sound and lighting, editing and scriptwriting must be woven into the fabric of computer and human considerations. Again the factors of production scheduling, casting and marketing must be included with the scheduling of programming and operational phases of the project. Certainly from a design perspective the development must be a combination of logical and creative processes. Thus the harnessing of human brainpower in the design is much more than a matter of logic.

The complexity of the design process highlights some current traffic jams. The traffic jam configuration in the design phase might manifest itself in questions of compatibility, priority, delivery or funding allocation. The selection of the 'right' medium is a major consideration in the development of materials, courseware and software for use with converging, integrated or multimedia technologies. As the choice expands the needs assessment becomes more critical than ever before. Historically projects incorporating 
convergent, integrated or multimedia have been 'idea' based, i.e. that have been developed under the criteria of exploring the potential of a new or dynamic medium, rather than on the basis of a learning need. The driving force in many cases has been the need to prove worthwhile a funding investment in the project. Learning needs assessment, while present in the initial briefings, has maintained a low priority in such developments.

In view of the requirement for such a range of expertise in projects and of the growing use of the technologies, we in education can no longer afford to minimise the role of a learning needs assessment. Further as the cost of development escalates and funding sources evaporate, the 'design' must become more comprehensive in its approach. In more practical terms, we currently have a considerable number of specialists with expertise in required areas. But the practicalities of institutional barriers such as competition for project funding, hamper the 'tourist guide' and 'information booth' network notions of bringing specialists together. The questions of competition and cooperation in education must be addressed if the cost of technology based learning development is not to remain prohibitive to the general education community.

Using already existing expertise is one alternative to the current traffic problem. A second, more long term alternative is the training of a greater number of both generalist and specialist designers. Few courses in tertiary education in Australia provide appropriate training for the design and production of materials for technology based instructional systems particularly in relation to converging, integrated or multimedia technologies. While experience may be an outstanding teacher, appropriate resource development into the 21st century can only be undertaken with an adequate number of resource personnel. With the push for training in business and industry and greater emphasis on technology based instruction in both the national and international marketplace in this sector the question of supply and demand of appropriately skilled designers becomes a major priority.

\section{Management traffic jams}

The design perspective is not the only area afflicted by traffic jams. Within the areas of technology implementation and management we also find numerous examples of both traffic hazards and traffic jams.

On the most simple level is the traffic jam caused by a lack of knowledge and understanding on the part of the user. Compact discs do physically fit into $51 / 2$ inch disc drives, but I doubt that the inventor intended them to be used that way.

Perhaps one of the more common 'traffic jams' is that caused by the congestion created through false expectations. Although technology is 
now capable of numerous tasks at exceptionally high speeds, they remain 'human driven'. In other words input and programming are still, at least for educational purposes, largely in the hands of the individuals designing, constructing or using the package. Despite the fact that technology is often seen, and sometimes presented, as the solution to every problem -- whatever the problem -- its success is dependent on a number of factors, any one of which might jeopardise the ultimate outcome.

A third type of traffic jam which might be placed in the domain of management is the case of too few machines and too many users. Although the number of computers in tertiary institutions is accelerating rapidly, the number of individuals wanting access to word processing, graphics and other computing facilities is growing disproportionately. Time limitations imposed in open access areas means a greater flow of through traffic but to the individual student the words "your time is up" can create havoc for the last minute assignment extension which expires that day.

The lack of support staff and appropriate allocation of funds and personnel for maintenance might be viewed as a fourth example of a management traffic jam. While the technology may be capable of many extremely complicated functions, it nevertheless requires 'human' input in order to remain operational or what has been described as 'userproof'. Current difficulties with viruses and other 'medical' conditions warrant even greater consideration of technical support. It might be argued that solutions to such difficulties are being found in the use of networks. These solutions however do not resolve the issues associated with the more sophisticated converging, integrated or multimedia technologies, designed primarily as stand alone packages.

\section{Getting the traffic moving}

What better way to spend one's time when detained by a traffic jam than to think of suggestions for possible solutions to the problems. The following suggestions are meant as starters to the thinking and planning process, rather than solutions in themselves.

In coming to terms with the convergence of technologies and the requirements of designers, management and learning, education must begin to put cooperation and teamwork high on the list of priorities. While undoubtedly, thanks to technology, the individual now has access to a much broader range of capabilities, access does not guarantee skill acquisition. Under the umbrella of cooperation we must also see greater employment of 'roadmaps'. Both governments and institutions must take a greater role in coordinating current undertakings in both research and implementation while maintaining 'academic and artistic' freedoms. 
Industry and business must become joint partners in these developments, not merely as a user of the end product, nor as a competitor in the field, but in cooperation with government and education in both development and implementation. Finally, in driving technology into the 21st century we must look towards achieving a balance between creativity and logic. We must allow the technology to be the passenger in the car.

\section{References}

Arwady, J. \& Gayeski, D. (1989). Using Video: Interactive and Linear Designs.

NJ: Educational Technology Publications.

Dede, C. (1990). The Coming Transformation of Educational Technology.

Keynote address presented to the Annual Convention of the

Association for Educational Communications and Technology,

Anahiem, February.

Please cite as: McNamara, S. (1990). Human-technology traffic jams (or harnessing human brainpower is more than a matter of logic). Australian Journal of Educational Technology, 6(1), 20-27.

http: / / www.ascilite.org.au/ajet/ajet6/mcnamara.html 